\title{
A Design of Terahertz Broadband Filters and its Effect in Eliminating Asymmetric Characteristics in Device Structures
}

\author{
Mei Zhu and Chengkuo Lee, Member, IEEE
}

\begin{abstract}
We report a straightforward way to design polarization-insensitive broadband bandstop filters in terahertz range by fabricating identical metal patterns on both sides of a dielectric substrate with one layer rotated $90^{\circ}$ to the other. The unit cells used in such designs are the well-studied split-ring-resonators, hence the frequency range of the stop band can be easily tuned by varying the dimensions of the unit cell structures. Through simulation analysis, we show that such fabrication method requires no alignment in lithography step and works with common dielectric substrates. Two types of substrates, 1-mm-thick quartz and $100-\mu \mathrm{m}$-thick polyethylene terephthalate, were used as a demonstration. The transmission level stayed below $12 \%$ for a stopband of $0.42-\mathrm{THz}$ frequency range. Lastly, we reasoned with simulation results that the proposed broadband filters give consistent performance regardless of the gap location in the unit cell, which will further extend their use in practical applications.
\end{abstract}

Index Terms-Broadband filter, metamaterials, polarization resonance, terahertz.

\section{INTRODUCTION}

$\mathbf{M}$ ETAMATERIALS are artificial composite materials designed to realize different optical properties and applications. They have attracted increasing research interest in the past decade for the many exotic phenomena they brought about, such as negative refractive index [1]-[3], perfect lens [4] and invisible cloaking [5], [6]. These unique properties and applications rely on the constituting structural elements to interact with external electromagnetic (EM) field and sustain resonances. One such element is a split ring resonator (SRR), first proposed and analyzed by Pendry et al. [7]. Such structures resonate to the incident EM wave at specific designed frequencies, and its first order resonant frequency can be easily calculated by modeling the structure as an inductor-capacitor $(L C)$ circuit [8]. Due to their simplicity, extensive studies have been done to form a complete picture regarding the application of SRRs in various designs of metamaterials, e.g. how the resonances relate

Manuscript received November 30, 2014; revised March 30, 2015; accepted May 5, 2015. Date of publication May 11, 2015; date of current version June 20, 2015. This work was supported in part by research grant MOE AcRF Tier 2-MOE2012-T2-2-154 under WBS R-263-000-A59-112 at the National University of Singapore, and the National Natural Science Foundation of China under Grant 61474078 at NUS (Suzhou) Research Institute, Suzhou, China.

The authors are with the Department of Electrical and Computer Engineering, National University of Singapore, 117581 Singapore, and also with the NUS Suzhou Research Institute, Suzhou, 215123 China (e-mail: zm_1805@hotmail.com; elelc@nus.edu.sg).

Color versions of one or more of the figures in this paper are available online at http://ieeexplore.ieee.org.

Digital Object Identifier 10.1109/JLT.2015.2432017 to structural dimensions [9], the coupling effect between the SRRs [10]-[16] and the influence on the resonances by the gap locations [17]-[19]. Moreover, their applications have been further extended by microelectromechanical systems (MEMS) technologies [20]-[26] and other tuning mechanisms [27]-[28].

On the other hand, there is an increasing demand for polarization-insensitive broadband filters to give more tolerance to manufacturing errors and allow multi-frequency operations [29]. SRRs have not been a desirable candidate for such use due to the narrowband and polarization-dependent characteristics of their resonances. Instead, isotropic patterns like metal patch [29], [30], cross patterns [31], [32] and closed rings [32], [33] were chosen as unit cell structures. These designs often have multiple layers stacked closely together and hence place stringent requirement on layer thickness [29] and alignment accuracy of each layer [30], [32]. Moreover, the stacking of lossy dielectric layers may also give rise to high transmission loss. In this work, we base our method on the well-studied SRR structures and report a simple method to fabricate polarization-insensitive broadband bandstop filters in $\mathrm{THz}$ frequency range. We show that our method places minimal requirement on substrate material type and thickness. Additionally, alignment accuracy in lithography steps is of little importance to device performance. Lastly, we also demonstrate through simulation that such a device is insensitive to the gap location in the SRR, making the design process even more flexible and hence broadening the scope of its applications.

\section{Simulation Development AND ExPERIMENTAl Details}

Our device consists of two metal layers fabricated on the two sides of a dielectric substrate. The metal patterns are identical except that one layer is rotated $90^{\circ}$ with respect to the other. The fabrication process flow is shown in Fig. 1. There are two types of substrates used in this work, 1-mm-thick quartz and $100-\mu$ m-thick polyethylene terephthalate (PET). The process was almost the same for both substrates except for the photoresist; AZ1512 was used for quartz and ultra- $i 123$ was used for PET [34], [35]. The fabrication starts with spincoating the respective photoresist on the substrate, at $2000 \mathrm{r} / \mathrm{min}$ for $1 \mathrm{~min}$ for AZ1512 and $800 \mathrm{r} / \mathrm{min}$ for $2.5 \mathrm{~min}$ for ultra- $i 23$ (step 1). After that, optical lithography was done to define negative SRR template on the photoresist (step 2). We then evaporated 200$\mathrm{nm}$ aluminum $(\mathrm{Al})$ onto the substrate using a thermal evaporator Edwards FL 400 (step 3). The first metal layer is completed with a final lift-off step by sonicating the sample in acetone for $2 \mathrm{~min}$ 


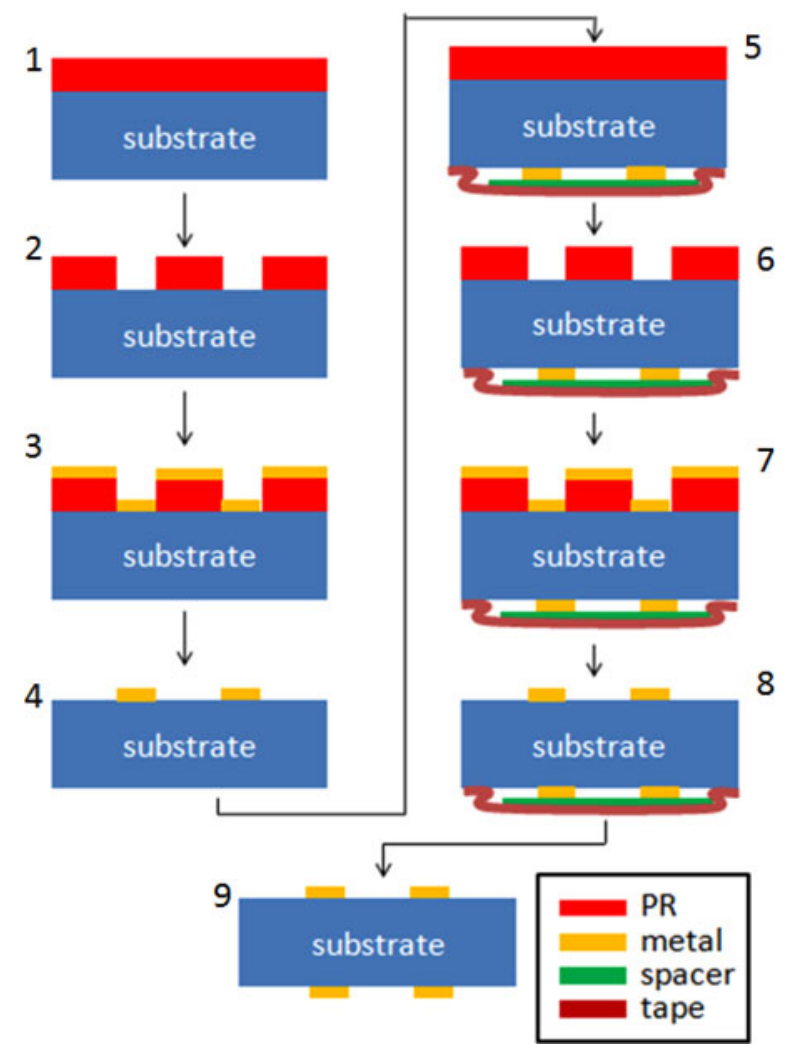

Fig. 1. Illustration of the process flow for fabricating the broadband filters which consist of two metal layers patterned on both sides of a dielectric substrate.

(step 4). The patterned side of the substrate was protected using a tape by enclosing the entire metal pattern inside with a spacer in between in order to prevent possible metal transfer onto the adhesive tape. Then, the process is repeated on the other side of the substrate (step 5-8). Eventually, removing the protective tape, the device was fabricated (step 9).

The metal patterns used in this work are modified SRRs with a split donut insert in the gap of conventional SRRs [8], as illustrated in Fig. 2(a). The period of the unit cell $(P)$ is $120 \mu \mathrm{m}$. The side length $(a)$ of the ring, line width $(l)$, outer diameter of the donut $(d)$ and the gap width $(g)$ are $80,6,30,4 \mu \mathrm{m}$, respectively. Depending on the distance of the donut to the side $(s)$, we designed symmetric and asymmetric unit cells for the proposed broadband filters, with $s$ being 25 and $8 \mu \mathrm{m}$, respectively. Fig. 2(b) and (c) show the unit cell of a symmetric and an asymmetric double-layered device, respectively. It is worth noting that in Fig. 2(c), the metal patterns on both sides are identical when both are viewed from the same direction. But if the device is to be fabricated, different masks should be used in step 2 and 6. The location of the donut in the two mask designs should be at the left bottom and right bottom of the square ring, respectively.

For comparison, we also studied and fabricated single-layered devices on both substrates as well following process steps 1 to 4. The EM responses of all devices were characterized using terahertz time domain spectroscopy (THz-TDS, TeraView TPS 3000) in transmission mode with normally incident $\mathrm{THz}$ wave.



(a)

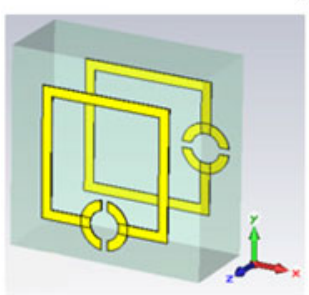

(b)



(c)
Fig. 2. (a) Schematic of the SRR pattern used to construct the proposed filters with dimension parameters labeled; (b) and (c) show the unit cells for symmetric and asymmetric double-layered devices.

TABLE I

DEVICES STUdied IN Simulation

\begin{tabular}{lcccc}
\hline \hline Devices & Unit cell & $\begin{array}{c}\text { Single/double } \\
\text { metal layer }\end{array}$ & substrate & metal \\
\hline Device 1 & symmetric & single & $\begin{array}{c}50-\mu \text { m-thick } \\
\text { quartz for all } \\
4 \text { devices }\end{array}$ & $\begin{array}{c}500 \text {-nm- } \\
\text { thick Al for } \\
\text { all } 4 \text { devices }\end{array}$ \\
Device 2 & symmetric & double & & \\
Device 3 & asymmetric & single & & \\
Device 4 & asymmetric & double & \\
\hline \hline
\end{tabular}

TABLE II

DEVICES FABRICATED AND CHARACTERIZED

\begin{tabular}{lcccc}
\hline \hline Devices & Unit cell & $\begin{array}{c}\text { Single/double } \\
\text { metal layer }\end{array}$ & substrate & metal \\
\hline Device A & symmetric & single & $\begin{array}{c}\text { 1-mm-thick } \\
\text { quartz for } \\
\text { A\&B }\end{array}$ & $\begin{array}{c}\text { 200-nm- } \\
\text { thick Al for } \\
\text { all devices }\end{array}$ \\
Device B & symmetric & double & single & $\begin{array}{c}100-\mu \text { m- } \\
\text { thick PET } \\
\text { for C\&D }\end{array}$ \\
Device D & symmetric & symmetric & double & \\
\hline \hline
\end{tabular}

A commercial FDTD solver, CST Microwave Studio was used to calculate the transmission spectrum as well as to find out electrical field distributions in the devices under various conditions. In simulation, $500-\mathrm{nm} \mathrm{Al}$ on $50-\mu$ m-thick quartz was used. The devices fabricated and simulated are further summarized in Tables I and II. 


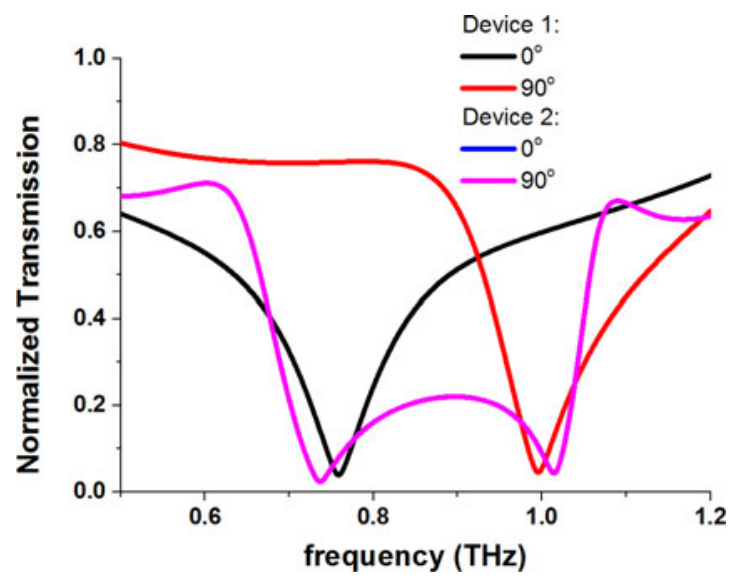

Fig. 3. Simulated transmission spectra for Device 1 and 2 with $0^{\circ}$ - and $90^{\circ}$ polarized incidence. For Device 2, the two spectra are identical and overlap.

\section{RESULTS AND DISCUSSIONS}

\section{A. Symmetric Devices}

Device 1 and 2 were first characterized using simulation software CST Microwave Studio. Two incident polarizations were studied in simulation, where one is parallel to the gap in the unit cell and the other is perpendicular to the gap. For the ease of description, they will be referred to as $0^{\circ}$ - and $90^{\circ}$-polarized incidence hereafter. Therefore, a $y$-polarized incidence for Device 2 is defined as $0^{\circ}$ with respect to the front layer and $90^{\circ}$ to the back layer, where the $x-y$ coordinate is defined in Fig. 2. For simplicity, only the polarization angle with respect to the front layer is stated when referring to double-layered devices.

The transmission spectrum for Device 1 and 2 under $0^{\circ}$ - and $90^{\circ}$-polarized incidences are presented in Fig. 3. It is evident that the single-layered Device 1 behaves as a polarization-sensitive narrowband bandstop filter like conventional SRR devices [8], as it couples to the external EM field at specific frequency depending on the incident polarization. More specifically, it resonates at 0.76 and $1.00 \mathrm{THz}$ for $\mathrm{a} 0^{\circ}$ - and $90^{\circ}$-polarized incidence, respectively. For Device 2, as the substrate thickness is greater than the attenuation length of the resonant electromagnetic modes [36], [37], there is negligible coupling between the two metal layers. When a $y$-polarized light transmits through Device 2, it effectively gets filtered by the front metal patterns as a $0^{\circ}$-polarized incidence at $0.76 \mathrm{THz}$, attenuates by the substrate, and gets filtered again by the back metal layer as a $90^{\circ}$-polarized incidence at $1.00 \mathrm{THz}$; while an $x$-polarized light experiences exactly the same in a reverse order. In other words, Device 2 is essentially the same as connecting two pieces of Device 1 in series with one rotated $90^{\circ}$ to the other, except for that it has only one dielectric layer and hence transmission loss is minimized. This explains why the stop band of Device 2 starts at $0.736 \mathrm{THz}$ and ends at $1.015 \mathrm{THz}$, extremely close to the two resonant frequencies of Device 1. On the other hand, as both filters do not have sharp transitions at band edges and the difference between their resonant frequencies is comparable to their $3-\mathrm{dB}$ bandwidths, the superposition of their spectra, i.e., the spectrum of Device 2

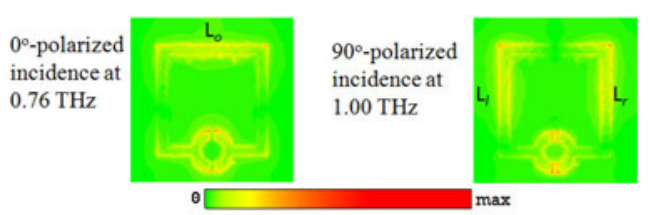

(a)

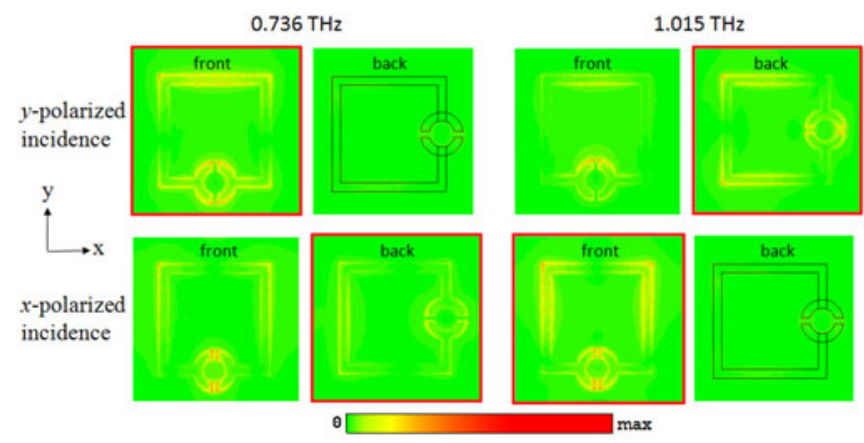

(b)

Fig. 4. Electrical field distributions at the resonant frequency for (a) Device 1 when illuminated by $0^{\circ}$ - and $90^{\circ}$-polarized incidences, as well as (b) at the beginning and end of the stopband for Device 2. The resonating metal layer in Device 2 is outlined in red.

has a broadband nature. Moreover, as the polarization azimuth of any randomly polarized light can be decomposed into $x$ and $y$ - direction, such a device is also polarization-insensitive. The fact that the frequency range of the stop band is essentially determined by the resonant frequencies of the narrowband single-layered device makes previous studies on SRR devices relevant and applicable to such broadband filters, which gives the design process a great advantage as compared to using other unconventional shapes as the unit cell.

The electrical field distributions for Device 1 at the two resonances and for Device 2 at the beginning and end of the stop band are also examined to provide further theoretical support to the design principle of such a double-layered broadband filter. The simulation results are shown in Fig. 4. Under differently polarized incidences, different parts of the metal pattern resonate to the external $\mathrm{EM}$ field. For $0^{\circ}$-polarized incidence, it is the side of the SRR containing the donut and the arm opposite $\left(\mathrm{L}_{0}\right)$ to the donut; for $90^{\circ}$-polarized incidence, it is the gap in the donut and the left and right sides of the SRR $\left(\mathrm{L}_{1}\right.$ and $\left.\mathrm{L}_{\mathrm{r}}\right)$, as shown in Fig. 4 (a). Following this, the previously discussed "series-connected filters" can be more intuitively viewed in Fig. 4(b). At $0.736 \mathrm{THz}$ with $y$-polarized incidence, the front metal layer resonates. No energy transmits to the back layer and hence it is not excited at all. At $1.015 \mathrm{THz}$, the front filter is less effective evidenced by the weaker electrical field excited in it. The transmitted energy is further filtered by the resonating back metal layer. The filtering processes for an $x$-polarized incidence is just the same but in reverse order, as expected.

As the simulation results suggest, there is little coupling between the two metal layers in Device 2, the thickness of the substrate and the alignment of the unit cells are therefore not important in fabricating a double-layered broadband filter. Following the steps described in Section II, we fabricated Device 


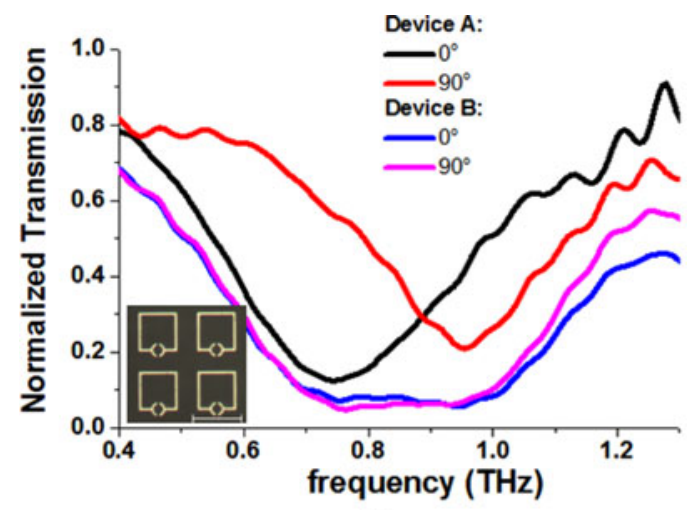

(a)

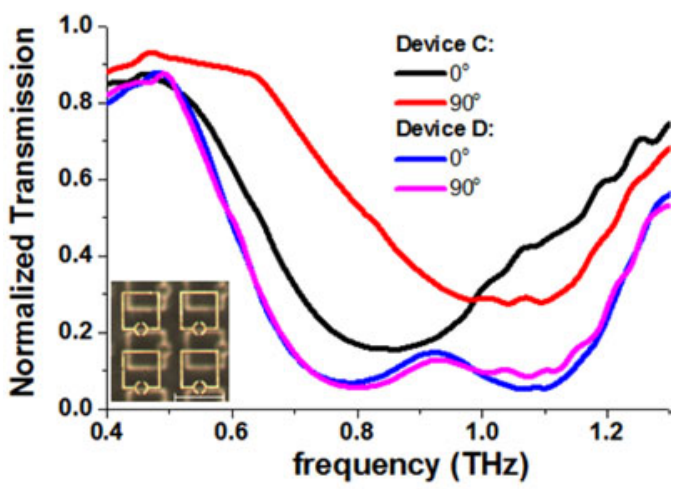

(b)

Fig. 5. Measured transmission spectrum of single- and double-layered devices on (a) 1-mm-thick quartz substrate and (b) $100-\mu \mathrm{m}$-thick PET substrate with $0^{\circ}$ and $90^{\circ}$ - incident polarization. The inserts are microscopic images of Device B and Device D respectively. The scale bars on both images are $100 \mu \mathrm{m}$.

A to D with no effort to align the unit cells in double-layered devices in the second lithography step. The transmission spectra of the fabricated devices were then measured using a THz-TDS, and normalized to that of a bare substrate with no metal patterns, shown in Fig. 5. The measured results agreed with simulation well. A polarization-insensitive stop band was achieved with both Device B and Device D, regardless of the substrate material and thickness. The transmission level stays below $12 \%$ from 0.68 to $1.01 \mathrm{THz}$ for Device B, and from 0.71 to $1.13 \mathrm{THz}$ for Device D.

\section{B. Asymmetric Device}

To study the effect of the gap location in the unit cell of the single and double-layered devices, we simulated the transmission spectra for Device 1 to 4 . In particular, we studied each entry in the Jones Matrix T [38], [39], where

$$
\boldsymbol{T}=\left(\begin{array}{c}
t_{x x} t_{x y} \\
t_{y x} t_{y y}
\end{array}\right)
$$

and $\boldsymbol{T}$ is a parameter linking the magnitude of the transmitted $\left(T_{\mathrm{i}}\right)$ and incident electrical field $\left(I_{\mathrm{i}}\right)$ in $x$ or $y$ direction in

$$
\left(\begin{array}{c}
T_{x} \\
T_{y}
\end{array}\right)=\boldsymbol{T}\left(\begin{array}{c}
I_{x} \\
I_{y}
\end{array}\right) .
$$

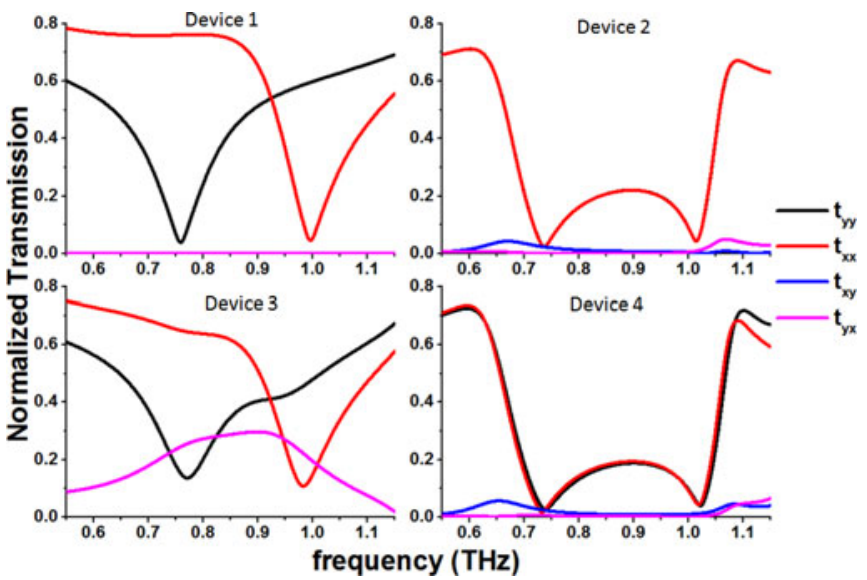

Fig. 6. Simulated transmission spectra of Device 1 to $4 . t_{\mathrm{ij}}$ stands for the transmission level of $j$-polarized light with an $i$-polarized incidence, with $x-y$ coordinate defined in Fig. 2.

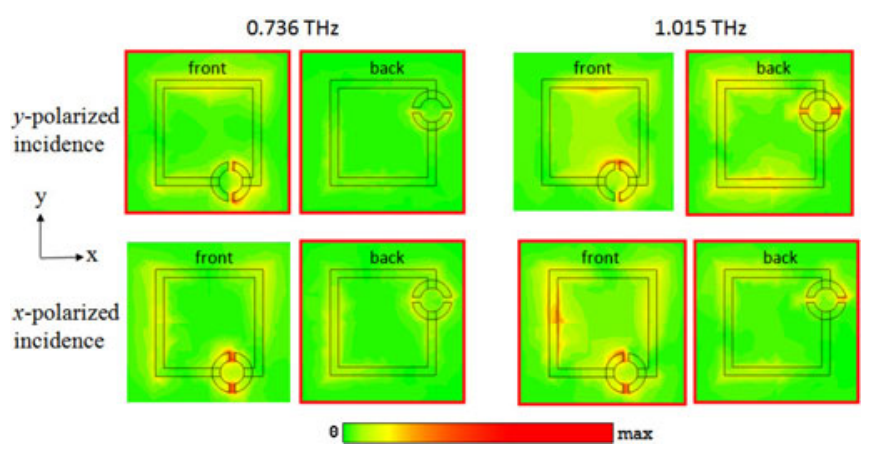

Fig. 7. Electrical field distributions in Device 4 at $0.736 \mathrm{THz}$ and $1.015 \mathrm{THz}$ when illuminated by $0^{\circ}$ - and $90^{\circ}$-polarized incidences with the resonating metal layer outlined in red.

The entries $t_{\mathrm{ij}}$ in Jones Matrix are parameters relating the incident $\left(E^{i n}\right)$ and the transmitted electrical fields $\left(E^{t r}\right)$ in terms of linearly polarized components in the expression [40]

$$
E_{i}^{t r}=t_{i j} E_{j}^{\text {in }}
$$

The simulation results of the transmission spectra are presented in Fig. 6. We found that for single layered devices, Device 3 with asymmetric unit cell is able to induce cross-polarized transmission, i.e., a significant non-zero $t_{\mathrm{xy}}$ and $t_{\mathrm{yx}}$; but such cross-polarized transmission is not seen in single layered Device 1 with symmetric unit cell, and is suppressed in both doublelayered devices. Device 2 and 4 have almost identical spectra despite the symmetry difference in their unit cells.

While the reason why asymmetric unit cell induces crosspolarized transmission is still under investigation, in this paper, we focus mainly on the interesting property of the doublelayered device that the transmission spectrum is invariant with the gap location in the designed SRR. To understand this, the electrical field distributions at the beginning and end of the stop band were also simulated for Device 4, as shown in Fig. 7. Since the single layered device is not an effective filter even at resonant frequencies with asymmetric unit cell, the transmitted light 
through the front metal layer changes its polarization state to orthogonal direction and excite the back layer as well. In Fig. 7, this is most obvious at $0.736 \mathrm{THz}$ for $y$-polarized incidence and at $1.015 \mathrm{THz}$ for $x$-polarized. The back layer that is not excited at these frequencies in Device 2 is now resonating in the same manner as the front layer. The $y$-polarized incident light is at $0^{\circ}$ to the front layer, and after polarization conversion, is at $0^{\circ}$ again to the back layer, therefore the donut and $\mathrm{L}_{\mathrm{o}}$ in both layers are excited. Similarly, under $x$-polarized incidence, both layers see $90^{\circ}$-polarized light, and $L_{\mathrm{l}}, L_{\mathrm{r}}$, and the gap in the donut are excited. These results show that in practical applications, symmetric and asymmetric SRRs are equally good candidates to be the unit cell and can be chosen at ease according to restrictions in the actual process.

\section{CONCLUSION}

In conclusion, we have reported a simple design method to fabricate polarization-insensitive broadband band stop filters in $\mathrm{THz}$ range. The design rules follow the well-studied SRR-based narrowband filters through which the stop band can be easily tuned by varying unit cell structural dimensions. The fabrication process poses minimal requirement on the substrate and alignment accuracy in lithography step. Moreover, the device is insensitive to the asymmetry in the unit cells. With these advantages, the cost and complexity in broadband filter design and fabrication can be significantly reduced. We have thus opened up a new door to filter designs that will greatly improve production efficiency.

\section{REFERENCES}

[1] V. G. Veselago, "The electrodynamics of substances with simultaneously negative values of $\varepsilon$ and $\mu$," Sov. Phys. Usp., vol. 10, no. 4, pp. 509-514, Jan. 1968.

[2] D. R. Smith, W. J. Padilla, D. C. Vier, S. C. Nemat-Nasser, and S. Schultz, "Composite medium with simultaneously negative permeability and permittivity," Phys. Rev. Lett., vol. 84, no. 18, pp. 4184-4187, May 2000.

[3] R. A. Shelby, D. R. Smith, and S. Schultz, "Experimental verification of a negative index of refraction," Science, vol. 292, no. 5514, pp. 77-79, 2001.

[4] J. B. Pendry, "Negative refraction makes a perfect lens," Phys. Rev. Lett., vol. 85, no. 18, pp. 3966-3969, 2000.

[5] J. B. Pendry, D. Schurig, and D. R. Smith, "Controlling electromagnetic fields," Science, vol. 312, no. 5781, pp. 1780-1782, 2006.

[6] D. Schurig, J. J. Mock, B. J. Justice, S. A. Cummer, J. B. Pendry, A. F. Starr, and D. R. Smith, "Metamaterial electromagnetic cloak at microwave frequencies," Science, vol. 314, no. 5801, pp. 977-980, 2006.

[7] J. B. Pendry, A. J. Holden, D. J. Robbins, and W. J. Stewart, "Magnetism from conductors and enhanced nonlinear phenomena," IEEE Trans. Microw. Theory Tech., vol. 47, no. 11, pp. 2075-2084, Nov. 1999.

[8] S. Linden, C. Enkrich, M. Wegener, J. Zhou, T. Koschny, and C. M. Soukoulis, "Magnetic response of metamaterials at 100 terahertz," Science, vol. 306, no. 5700, pp. 1351-1353, Nov. 2004.

[9] A. K. Azad, A. J. Taylor, E. Smirnova, and J. F. O'Hara, "Characterization and analysis of terahertz metamaterials based on rectangular split-ring resonators," Appl. Phys. Lett., vol. 92, no. 1, pp. 011119-1-011119-3, Jan. 2008.

[10] N. Liu, S. Kaiser, and H. Giessen, "Magnetoinductive and electroinductive coupling in plasmonic metamaterial molecules," Adv. Mater, vol. 20, pp. 4521-4525, Oct. 2008.

[11] N. Liu, H. Liu, S. Zhu, and H. Giessen, "Stereometamaterials," Nature Photon., vol. 3, no. 4, pp. 157-162, Mar. 2009.

[12] T. Q. Li, H. Liu, T. Li, S. M. Wang, J. X. Cao, Z. H. Zhu, Z. G. Dong, S. N. Zhu, and X. Zhang, "Suppression of radiation loss by hybridization effect in two coupled split-ring resonators," Phys. Rev. B, vol. 80, no. 11, pp. 115113-1-115113-6, Sep. 2009.

[13] J. García-García, F. Martín, J. D. Baena, R. Marqués, and L. Jelinek, "On the resonances and polarizabilities of split ring resonators," J. Appl. Phys., vol. 98, no. 3, pp. 033103-1-033103-9, Aug. 2005.

[14] N. Liu, H. Guo, L. Fu, H. Schweizer, S. Kaiser, and H. Giessen, "Electromagnetic resonances in single and double split-ring resonator metamaterials in the near infrared spectral region," Phys. Stat. Sol. (b), vol. 244, no. 4, pp. 1251-1255, Mar. 2007.

[15] H. Guo, N. Liu, L. Fu, T. P. Meyrath, T. Zentgraf, H. Schweizer, and H. Giessen, "Resonance hybridization in double split-ring resonator metamaterials," Opt. Exp., vol. 15, no. 19, pp. 12095-12101, Sep. 2007.

[16] M. Zhu, Y. Lin, and C. Lee, "Coupling effect combined with incident polarization to modulate double split-ringresonator in terahertz frequency range," J. Appl. Phys., vol. 116, no. 17, pp. 173106-1-173106-7, Nov. 2014

[17] V. A. Fedotov, M. Rose, S. L. Prosvirnin, N. Papasimakis, and N. I. Zheludev, "Sharp trapped-mode resonances in planar metamaterials with a broken structural symmetry," Phys. Rev. Lett., vol. 99, no. 14, pp. 147401-1-147401-4, Oct. 2007.

[18] R. Singh, I. A. I. Al-Naib, M. Koch, and W. Zhang, "Asymmetric planar terahertz metamaterials," Opt. Exp., vol. 18, no. 12, pp. 13044-13050, Jun. 2010.

[19] Y. Yang, R. Huang, L. Cong, Z. Zhu, J. Gu, Z. Tian, R. Singh, S. Zhang, J. Han, and W. Zhang, "Modulating the fundamental inductive-capacitive resonance in asymmetric double-split ring terahertz metamaterials," Appl. Phys. Lett., vol. 98, no. 12, pp. 121114-1-121114-3, Mar. 2011.

[20] W. M. Zhu, A. Q. Liu, X. M. Zhang, D. P. Tsai, T. Bourouina, J. H. Teng, X. H. Zhang, H. C. Guo, H. Tanoto, T. Mei, G. Q. Lo, and D. L. Kwong, "Switchable magnetic metamaterials using micromachining processes," Adv. Mater., vol. 23, no. 15, pp. 1792-1796, Apr. 2011.

[21] Y. H. Fu, A. Q. Liu, W. M. Zhu, X. M. Zhang, D. P. Tsai, J. B. Zhang, T. Mei, J. F. Tao, H. C. Guo, X. H. Zhang, J. H. Teng, N. I. Zheludev, G. Q. Lo, and D. L. Kwong, "A micromachined reconfigurable metamaterial via reconfiguration of asymmetric split-ring resonators," Adv. Funct. Mater., vol. 21, no. 18, pp. 3589-3594, Sep. 2011.

[22] Y. Lin, Y. Qian, F. Ma, Z. Liu, P. Kropelnicki, and C. Lee, "Development of stress-induced curved actuators for a tunable $\mathrm{THz}$ filter based on double split-ring resonators," Appl. Phys. Lett., vol. 102, no. 11, pp. 111908-1111908-5, Mar. 2013.

[23] F. Ma, Y. Lin, X. Zhang, and C. Lee, "Tunable multiband terahertz metamaterials using a reconfigurable electric split-ring resonator array," Light Sci. Appl., vol. 3, no. e171, pp. 1-8, May 2014.

[24] Y. Lin, F. Ma, and C. Lee, "Three-dimensional movable metamaterial using electric split-ring resonators," Opt. Lett., vol. 38, no. 16, pp. 3126-3128, Aug. 2013.

[25] H. Tao, A. C. Strikwerda, K. Fan, W. J. Padilla, X. Zhang, and R. D. Averitt, "Reconfigurable terahertz metamaterials," Phys. Rev. Lett., vol. 103, no. 14, pp. 147401-1-147401-4, Oct. 2009.

[26] Y. Lin, C. Huang, and C. Lee, "Reconfiguration of resonance characteristics for terahertz U-shape metamaterial using MEMS mechanism," IEEE J. Sel. Top. Quantum Electron., vol. 21, no. 4, pp. 2700207-1-2700207-7, Jul. 2015 .

[27] H. Chen, W. J. Padilla, J. M. O. Zide, A. C. Gossard, A. J. Taylor, and R. D. Averitt, "Active terahertz metamaterial devices," Nature, vol. 444, pp. 597-600, Nov. 2006.

[28] H. Chen, J. F. O'Hara, A. K. Azad, A. J. Taylor, R. D. Averitt, D. B. Shrekenhamer, and W. J. Padilla, "Experimental demonstration of frequency-agile terahertz metamaterials," Nature Photon., vol. 2, pp. 295-298, Apr. 2008.

[29] J. Han, J. Gu, X. Lu, M. He, Q. Xing, and W. Zhang, "Broadband resonant terahertz transmission in a composite metal-dielectric structure," Opt. Exp., vol. 17, no. 19, pp. 16527-16534, Sep. 2009.

[30] J. Zhu, Z. Ma, W. Sun, F. Ding, Q. He, L. Zhou, and Y. Ma, "Ultrabroadband terahertz metamaterial absorber," Appl. Phys. Lett., vol. 105, no. 2, pp. 021102-1-021102-4, Jul. 2014.

[31] Y. Q. Ye, Y. Jin, and S. He, "Omnidirectional, polarization-insensitive and broadband thin absorber in the terahertz regime," J. Opt. Soc. Amer. B, vol. 27 , no. 3, pp. 498-504, Mar. 2010.

[32] L. Liang, B. Jin, J. Wu, Y. Huang, Z. Ye, X. Huang, D. Zhou, G. Wang, X. Jia, H. Lu, L. Kang, W. Xu, J. Chen, and P. Wu, "A flexible wideband bandpass terahertz filter using multi-layer metamaterials," Appl. Phys. B, vol. 113, pp. 285-290, May 2013.

[33] Z. Li and Y. J. Ding, "Terahertz broadband-stop filters," IEEE J. Sel. Top. Quantum Electron., vol. 19, no. 1, pp. 8500705-1-8500705-5, Jan. 2013. 
[34] M. Zhu, L. Zhou, B. Li, M. K. Dawood, G. Wan, C. Q. Lai, H. Cheng, K. C. Leong, R. Rajagopalan, H. P. Too, and W. K. Choi, "Creation of nanostructures by interference lithography for modulation of cell behavior," Nanoscale, vol. 3, pp. 2723-2729, Apr. 2011.

[35] M. Zhu, B. Li, and W. K. Choi, "Fabrication of nanostructures on polyethylene terephthalate substrate by interference lithography and plasma etching," J. Nanosci. Nanotechnol., vol. 13, no. 8, pp. 5474-5480, Aug. 2013.

[36] F. Miyamaru, M. W. Takeda, and K. Taima, "Characterization of terahertz metamaterials fabricated on flexible plastic films: Toward fabrication of bulk metamaterials in terahertz region," Appl. Phys. Exp., vol. 2, no. 4, pp. 042001-1-042001-3, Mar. 2009.

[37] F. Miyamaru, S. Kuboda, K. Taima, K. Takano, M. Hangyo, and M. W. Takeda, "Three-dimensional bulk metamaterials operating in the terahertz range," Appl. Phys. Lett., vol. 96, no. 8, pp. 081105-1-081105-3, Feb. 2010.

[38] R. C. Jones, "A new calculus for the treatment of optical systems," J. Opt. Soc. Amer, vol. 27, no. 7, pp. 488-493, Jul. 1941.

[39] C. Huang, Y. Feng, J. Zhao, Z. Wang, and T. Jiang, "Asymmetric electromagnetic wave transmission of linear polarization via polarization conversion through chiral metamaterial structures," Phys. Rev. B, vol. 85, pp. 195131-1-195131-5, May 2012.

[40] R. Singh, E. Plum, C. Menzel, C. Rockstuhl, A. K. Azad, R. A. Cheville, F. Lederer, W. Zhang, and N. I. Zheludev, "Terahertz metamaterial with asymmetric transmission," Phys. Rev. B, vol. 80, no. 15, pp. 153104-1153104-4, Oct. 2009.

Mei Zhu received the B.Eng. and Ph.D. degrees from the Department of Electrical and Computer Engineering, National University of Singapore, Singapore, in 2009 and 2014, respectively.

She is currently a Research Fellow in the same department. Her research interests include microfluidic chips for cell studies and applications of metamaterial devices.
Chengkuo Lee (S'93-M'96) received the M.S. degree in materials science and engineering from the National Tsing Hua University, Hsinchu, Taiwan, in 1991, the M.S. degree in industrial and system engineering from Rutgers University, New Brunswick, NJ, USA, in 1993, and the Ph.D. degree in precision engineering from the University of Tokyo, Tokyo, Japan, in 1996.

He was a Foreign Researcher at the Nanometerscale Manufacturing Science Laboratory, Research Center for Advanced Science and Technology, University of Tokyo, from 1993 to 1996. He had also worked with the Technical Engineering Laboratory, Advanced Industrial Science and Technology, Ministry of International Trade and Industry of Japan, as a Japan Science and Technology Research Fellow in 1996. Thereafter, he was a Senior Research Staff Member at the Microsystems Laboratory, Industrial Technology Research Institute, Hsinchu. In September 1997, he joined the Metrodyne Microsystem Corporation, Hsinchu, and established the Microelectromechanical Systems (MEMS) Device Division and the first micromachining laboratory for commercial purposes in Taiwan. He was the Manager of the MEMS Device Division between 1997 and 2000. He was an Adjunct Assistant Professor at the Department of Electrophysics, National Chiao Tung University, Hsinchu, in 1998, and an Adjunct Assistant Professor at the Institute of Precision Engineering, National Chung Hsing University, Taichung, Taiwan, from 2001 to 2005. He cofounded Asia Pacific Microsystems, Inc. (APM), Hsinchu, in August 2001, and he became the Vice President of R\&D; then, later till the end of 2005, he became the Vice President of the optical communication business unit and the Special Assistant to the Chief Executive Officer in charge of international business and technical marketing for MEMS foundry service at APM, Inc., one of the top 30 MEMS manufacturers in the world in 2004. From 2006 to 2009, he was a Senior Member of Technical Staff at the Institute of Microelectronics, A*STAR, Singapore. He is currently an Associate Professor at the Department of Electrical and Computer Engineering, National University of Singapore, Singapore. $\mathrm{He}$ has contributed more than 250 international conference papers and extended abstracts, 180 peer-reviewed international journal articles, and nine U.S. patents in the MEMS, NEMS, metamaterials, nanophotonics, and nanotechnology fields. 